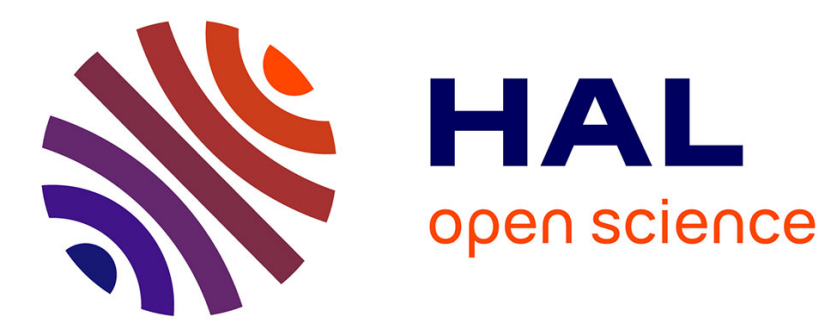

\title{
Throughput-Aware RRHs Clustering in Cloud Radio Access Networks
}

Nazih Salhab, Rana Rahim, Rami Langar

\section{To cite this version:}

Nazih Salhab, Rana Rahim, Rami Langar. Throughput-Aware RRHs Clustering in Cloud Radio Access Networks. 2018 Global Information Infrastructure and Networking Symposium (GIIS), Oct 2018, Thessaloniki, Greece. pp.1-5, 10.1109/GIIS.2018.8635647 . hal-02266995

\section{HAL Id: hal-02266995 \\ https://hal.science/hal-02266995}

Submitted on 29 May 2020

HAL is a multi-disciplinary open access archive for the deposit and dissemination of scientific research documents, whether they are published or not. The documents may come from teaching and research institutions in France or abroad, or from public or private research centers.
L'archive ouverte pluridisciplinaire HAL, est destinée au dépôt et à la diffusion de documents scientifiques de niveau recherche, publiés ou non, émanant des établissements d'enseignement et de recherche français ou étrangers, des laboratoires publics ou privés. 
THIS IS AN AUTHOR-CREATED POSTPRINT VERSION, A.K.A. ACCEPTED VERSION, AND NOT THE PUBLISHED VERSION AS IT MIGHT BE DOWNLOADED FROM IEEE XPLORE, TAKING INTO CONSIDERATION REVIEWERS COMMENTS.

Below are some Frequently Asked Questions (FAQs) (Excerpt from IEEE Author FAQ):

https://www.ieee.org/content/dam/ieee-org/ieee/web/org/pubs/author_faq.pdf

\section{Originality of Content}

- Does IEEE consider an author posting her paper on preprint servers or on her company's web sites to be a form of prior publication, which may then disqualify the paper from further editorial consideration?

No. IEEE policy allows an author to submit previously posted papers to IEEE publications for consideration as long as she is able to transfer copyright to IEEE, i.e., she had not transferred copyright to another party prior to submission.

\section{Authors' Rights to Post Accepted Versions of Papers}

- Can an author post his IEEE copyrighted paper on his personal or institutions' servers? Yes. An author is permitted to post his IEEE copyrighted paper on his personal site and his institution's server, but only the accepted version of his paper, not the published version as might be downloaded from IEEE Xplore.

- Can an author post his manuscript on a preprint server such as TechRxiv or ArXiv? Yes. The IEEE recognizes that many authors share their unpublished manuscripts on public sites. Once manuscripts have been accepted for publication by IEEE, an author is required to post an IEEE copyright notice on his preprint. Upon publication, the author must replace the preprint with either 1 ) the full citation to the IEEE work with Digital Object Identifiers (DOI) or a link to the paper's abstract in IEEE Xplore, or 2) the accepted version only (not the IEEEpublished version), including the IEEE copyright notice and full citation, with a link to the final, published paper in IEEE Xplore.

Disclaimer:

This work was accepted for publication in the IEEE. Final version after revision is accessible through: https://ieeexplore.ieee.org/Xplore/home.jsp

\section{IEEE Xplore}

Copyright:

CIEEE. Personal use of this material is permitted. Permission from IEEE must be obtained for all other uses, in any current or future media, including reprinting/republishing this material for advertising or promotional purposes, creating new collective works, for resale or redistribution to servers or lists, or reuse of any copyrighted component of this work in other works. 


\title{
Throughput-Aware RRHs Clustering in Cloud Radio Access Networks
}

\author{
Nazih Salhab*§, Rana Rahim ${ }^{\ddagger}$, and Rami Langar* \\ $\S$ Faculty of Engineering, LTRM-EDST, Lebanese University, Lebanon \\ $\ddagger$ Faculty of Science, LTRM-EDST, Lebanese University, Lebanon \\ * University Paris-Est, LIGM-CNRS UMR 8049, UPEM, F-77420, Marne-la-Vallée, France \\ E-mails: nazih.salhab@u-pem.fr; rana.rahim@ul.edu.lb; rami.langar@u-pem.fr
}

\begin{abstract}
Cloud-Radio Access Network (C-RAN) is an attractive solution to Mobile Network Operators. Firstly, C-RAN leverages the effect of pooling multiple Baseband Units (BBUs) to offer centralized processing resources while hosting them on cloud. This results in multiple benefits ranging from statistical multiplexing gains, to energy efficiency. Secondly, C-RAN allows deploying Remote Radio Heads (RRHs) in proximity of end-users allowing exploiting Inter-Cell Interference Cancellation (ICIC) to maximize throughput by coordinating multiple RRHs. In this context, we propose, in this paper, a new throughput-aware RRHs clustering method for C-RAN that maximizes the throughput for end-users, while meeting multiple constrained resources on BBUs. Our approach consists of two stages: First, individual throughput value and requirements of each RRH are calculated taking into account the Signal-to-Interference-plus-Noise Ratio (SINR) values and the distance between RRHs and users. Then, they are included into a k-dimensional Multiple-Choice Knapsack Problem (k-MCKP) subject to several constraints in terms of required resources in order to form RRHs clusters that maximize the global throughput. Simulation results demonstrate the good performance of our proposal in terms of end-users throughput, spectral efficiency and execution time, when compared with the optimal solution and the basic strategy using no-clustering scenarios.
\end{abstract}

Index Terms-C-RAN, BBU Pool, RRH Cluster, k-MCKP, CoMP

\section{INTRODUCTION}

With the introduction of social media and smart phones, endusers became more and more inseparable from their phones and continuously generating and consuming contents, through their data-hungry devices. In 4G, LTE allowed Mobile Network Operators (MNO) to offer Mobile Broadband (MBB) with download speeds going up to 300 Mbps. 5G advocates a mechanism called "Slicing" applicable to both Core Network (CN) and Radio Access Network (RAN). Slicing enables MNO to decompose their network architectures into virtual networks (slices). Each slice is optimized to serve different set of competing constraints in terms of throughput, latency, reliability, energy efficiency among other constraints. Enhanced Mobile Broadband (eMBB), also referred to as Extreme Mobile Broadband (xMBB) is a class of $5 \mathrm{G}$ Slicing for RAN where the focus is confined to throughput and mobility. In eMBB, the throughput is planned to be higher than one Gbps and the mobility is up to $500 \mathrm{Km} / \mathrm{h}$. Such eMBB allows catering higher data-rate demands for end-users enabling them to constantly generate andconsume data even when they are in extreme conditions (high velocity and high demands) [1].

Cloud-RAN (C-RAN) is an attractive solution to MNO [2]. It saves CAPEX by leveraging the benefits of Base Stations (BSs) components' distribution and functionalities' split in eNodeB

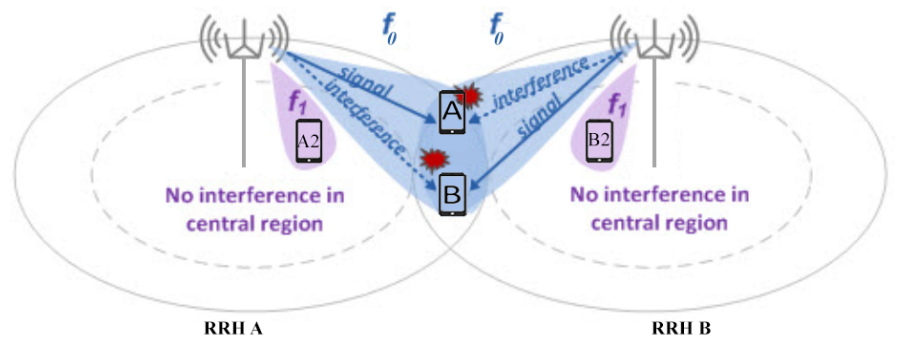

Fig. 1. Inter Cell Interference Cancellation Principle

(eNB). Specifically, the Distributed Base Station (DBS) is decomposed into BaseBand Unit (BBU) for baseband processing and Remote Radio Head (RRH) for radio and physical layer functionality [3]. Knowing that not all BBU resources are constantly used by a certain DBS eNB, BBU Pooling allows usage maximization in three folds. Indeed, BBU pooling results in decreasing the number of BBUs by gaining from statistical multiplexing.It also increases resiliency against failure (no single point of failure). In addition, it allows to leverage coordination to exploit interference in the favor of throughput increase. BBU Pooling is especially feasible with the advents of Network Function Virtualization (NFV) [4], which allows implementing vendor-based hardware BBU by a software-based Virtual BBU (vBBU). Not only does the grouping take place on BBU side, to save the CAPEX but also, RRHs are grouped as "RRH Clusters".

RRHs clustering results in mitigated interference among RRHs or even better, an increase of throughput in case of exploited interference. This mechanism is called Inter-Cell Interference Cancellation (ICIC) [3] as depicted in Fig. 1.

ICIC can take place in one of the following three forms. These forms are (1) Coordinated Scheduling and Coordinating Beam-forming of RRHs (CS/CB), (2) Dynamic Point Selection (DPS) to favor the best RRH to serve the end-user or (3) Joint Transmission (JT) from multiple RRHs to a user. Coherent JT achieves simultaneous power and multiplexing gains. The coordination mechanism among RRHs by the BBUs is called Coordinated Multi-Point (CoMP) [5].

To this end, we propose a two-stage approach. First, the throughput value and weights of the impact of mapping each RRH on BBU processing resources are calculated. Next, such inputs are included into a k-dimensional Multiple-Choice Knapsack Problem (k-MCKP), which is then solved using a simple yet efficient heuristic. A "k-MCKP" is computationally harder than one dimension Multiple-Choice Knapsack Problem 
(MCKP) [6]. MCKP is a particular case of K-MCKP where $\mathrm{k}$ is equal to 1 . Our goal is to select the RRHs cluster that maximizes the throughput for end-users while meeting multiple constraints on BBUs resources.

It is worth noting that, although, maximization of the cluster to include the biggest number of RRHs reduces the inter-cluster handover, this has the pitfall of increased BBU resources usage and dramatic increase of load on front-haul links. Such trivial solution is typically rejected due to the imposed constraints on the BBUs. Our contributions are summarized as follows:

- Previous implementations of RRHs Clustering consider fixed size single BBU when clustering the RRHs. Our work considers multiple BBU pool with several constraints as based on real hardware implementation from telecommunications equipments' suppliers [7].

- We validate our problem formulation by means of simulation and show the effectiveness of our proposal compared to the optimal solution as well as the basic strategy using no-clustering scenarios.

The remainder of this paper is organized as follows. Section II presents an overview of related works. Section III describes the system model and formulates the traffic aware clustering as k-MCKP and discusses the complexity of the optimal and heuristic solutions. Section IV elaborates the performance evaluation and the simulation environment and discusses the results. Section V concludes this paper.

\section{RELATED WORKS}

RRHs Clustering problem in C-RAN has incited considerable research efforts in the last few years. We classify the reviewed papers described in this section into two categories. The first category covers papers that handled the RRHs Clustering problem. The second category covers papers that treated MCKP problem in other applications. Papers in the first category are [8], [9], [10], [11] and [12]. Authors in [8] have handled RRHs Clustering problem from interference-awareness perspective. They have proposed an interference aware clustering algorithm in C-RAN based on set partitioning problem aiming to minimize ICIC using optimal and heuristic approaches. Authors in [9] formulated the RRHs Clustering problem as a coalition formation game to optimize throughput, power consumption and handover frequency. Authors in [10] worked on optimal and heuristic solutions to RRHs Clustering, while considering re-association between BBUs and RRHs. Although all of these papers showed considerable improvement on treating the RRHs Clustering problem, they did consider a fixed size group on BBU side, resulting in a "Bin packing problem". The fixed size assumption on BBU is not the case a fortiori in C-RAN due to the usage of cloud. In fact, in the C-RAN context, BBUs are pooled together to offer a higher processing capability than a single BBU. In addition, when stored on the cloud, BBUs leverage easiness of elasticity in cloud computing [4]. Authors in [11] considered the BBU-RRH problem from quality of service (QoS) perspective and adopted a genetic algorithm (GA) and Discrete Particle Swarm Optimization (DPSO) approach to solve it. However, as opposed to our proposal, the objective was not to maximize the global throughput. Authors in [12] considered the problem of BBU-RRH assignment as Multiple Knapsack Problem (MKP) but assumed one single constraint on the BBU pool which is not the case in real life where multiple constraints bound the capacity of the BBUs as elaborated in next section. Indeed, differently from [12], we considered multiple constraints on the BBU pool. Papers in the second category are [13] and [14]. They handled different problems using k-dimensional MCKP modeling. In fact, authors in [13] designed a resource aware architecture for adaptable and distributed systems using k-MCKP. Authors in [14] adopted the $\mathrm{k}$-dimensional MCKP for cognitive radio applications. Such papers used the k-MCKP in different context and did not cover details about the simulation method used. In our work, thorough details about the simulation are elaborated in the CRAN context.

\section{SYSTEM MODEL AND PROBLEM FORMULATION}

\section{A. System Model}

In this paper, we focus on clustering RRHs together in a way to maximize the system throughput provided that each cluster is parented to a BBU pool. For the implementation of the k-MCKP-based clustering approach, the choice of cluster in regard to the epoch of time during which the cluster is changed is not instantaneous. In fact, the cluster is not supposed to change in a matter of seconds or minutes, otherwise, this would trigger massive reconfigurations which are costly on the fronthaul interface. Typically, based on current telecommunication equipments' suppliers vendors, the performance measurement Key Performance Indicators (KPI) collected from field radio nodes (RRHs) are of 15 minutes granularity. Occasionally, the granularity is re-tuned on demand and for a limited period to 5 minutes resolution in order to avoid overloading the Operation Support Subsystem (OSS). Also, based on seasonality of traffic trends, the clustering scheme is typically suggested to be carried out at a scale of hours. Without loss of generality, we assume that each BBU capacity is constrained by the following five constraints [7]:

- Maximum number of RRHs that depends on (nT).(nR) beam-forming or MIMO configuration, where $n T, n R$ denote the numbers of transmit and receive antennas respectively.

- Maximum throughput per RRH that is the downlink data rate at the Medium Access Control (MAC) layer for the typical 5/10/20 MHz bandwidth according to the Carrier Aggregation (CA) scheme.

- Maximum throughput per BBU that is the sum of uplink and downlink data rates at the MAC layer.

- Maximum number of users in the Radio Resource Control mode (RRC_CONNECTED) in a BBU. A user is in RRC_CONNECTED when an RRC connection is established.

- Data Radio Bearer (DRB) per User Equipment (UE).

We model the arrival of user's equipment (UE) by a Poisson Point Process (PPP) [15], where UEs are spread and attached to RRHs. Each user demand is function of its usage represented by the required bandwidth and is expressed in number of Physical Resource Blocks (PRBs). A PRB is the smallest element of resource allocation assigned by the eNB scheduler.

Each RRH is characterized by a certain value contribution $(v)$ to the throughput. To calculate the throughput, we used the theorem of Shannon-Hartley that ties the channel capacity to the bandwidth and Signal-to-Noise-and-Interference Ratio (SINR).

$$
\text { ChannelCapacity }=B \log _{2}(1+S I N R)
$$


$B$ is channel bandwidth in Hertz. The channel capacity provides an upper bound to the peak throughput allowed in this channel in bits per second. The simulation depicted in Fig. 2 shows the achieved throughput in regards to channel capacity. Our interference model, and its parameters are based on the 3GPP technical specification [15]. The SINR is given by :

$$
\operatorname{SIN}_{r, k}^{(u)}=\frac{p_{r, k}^{(u)} \cdot g_{r, k}^{(u)}}{N_{0}+\sum_{s \neq r} \sum_{v \neq u} p_{s, k}^{(u)} \cdot g_{s, k}^{(u)}}
$$

Where $p$ is the signal power and $g$ is the channel gain from RRH $r$ towards UE $u$ on PRB $k$. The noise power is denoted as $N_{0}$. The received power is calculated from the transmitted power subtracted by the path loss expressed in decibels [15]. The path loss is computed as follows:

$$
L_{F S}=92.45+20 \log _{10}(f)+20 \log _{10}(d)
$$

Where $L_{F S}$ is the path loss in free space (in $\mathrm{dB}$ ). The frequency $f$ is expressed in gigahertz, and the distance between transmitter and receiver $d$ is expressed in kilometers.

\section{B. Problem Formulation}

Let us first introduce a binary variable $x_{i j}$ to indicate whether the RRH $j$ is assigned to the cluster $i$ or not. We denote by $v_{j}$ the throughput value of RRH $j$. Each RRH $j$ is also characterized by some weights $w_{j}^{(k)}$ that are the consumed portions of resources from the BBU pool $i$. Weights are functions of the traffic profiles being served by these RRHs.The two indexes $n$ and $m$ denote the number of RRHs and number of BBU pools respectively. Our objective is to form a cluster of RRHs in such a way that the total throughput is maximized, while considering the multiple resource constraints. Each RRH $j$ of cluster $i$ has the non-negative throughput value $v_{i j}$, and requires resources from the BBU pool that has limited processing capacities represented by the weight vector $W_{i j}=\left(w_{i j}^{(1)}, w_{i j}^{(2)}, \ldots, w_{i j}^{(K)}\right)$, where $K$ denotes the number of resource constraints on the BBU pool. Note that $m$ needs to be lower or equal than the total number of BBUs in the network, as the trivial mapping of RRH to $\mathrm{BBU}$ is 1 to 1 . The k-MCKP is constrained by a constraint vector $C=\left(C^{(1)}, C^{(2)}, \ldots, C^{(k)}, \ldots, C^{(K)}\right)$ characterizing the upper bound of available BBU resources. Our clustering problem is formulated as k-MCKP, as follows:

$$
\begin{aligned}
& \underset{x}{\operatorname{maximize}} \quad z=\sum_{i=1}^{m} \sum_{j=1}^{n} v_{j} x_{i j} \\
& \text { subject to } \\
& \qquad \sum_{j=1}^{n} w_{j}^{(k)} x_{i j} \leq C_{i}^{(k)} ; i \in\{1, \ldots, m\} ; k \in\{1, \ldots, K\} \\
& \quad \sum_{i=1}^{m} x_{i j} \leq 1 ; j \in\{1, \ldots, n\} \\
& x_{i j} \in\{0,1\} ; i \in\{1, \ldots, m\}, j \in\{1, \ldots, n\}
\end{aligned}
$$

Equation (4a) is the objective function to maximize. The set of equations (4b) specifies the $\mathrm{K}$ constraints on resources. Equation (4c) is the exclusivity constraint to make sure that each RRH is parented to one BBU pool only.
The formulated k-MCKP is a Non-deterministic Polynomialtime Hard (NP-Hard) combinatorial optimization problem [16] [17]. We propose a simple yet efficient heuristic to solve the k-MCKP problem providing values close to optimum ones with noticeable gain in terms of simplicity and rapidity for realistic input size. The heuristic listed in Algorithm 1 works as follows:

Sort constraints on BBU pool in increasing order to identify the first bottleneck. The index for this first bottleneck is denoted $k^{*}$. Disregard any RRH not satisfying all the constraints all together. Calculate the efficiency that is the throughput value of each RRH divided by the constraint value in regard with first priority bottleneck $\left(C^{(k *)}\right)$. Sort Efficiencies in decreasing order. Get the RRH of biggest efficiency in the cluster. Try to include additional RRH if they satisfy the constraints. Get the throughput value of the formed cluster.

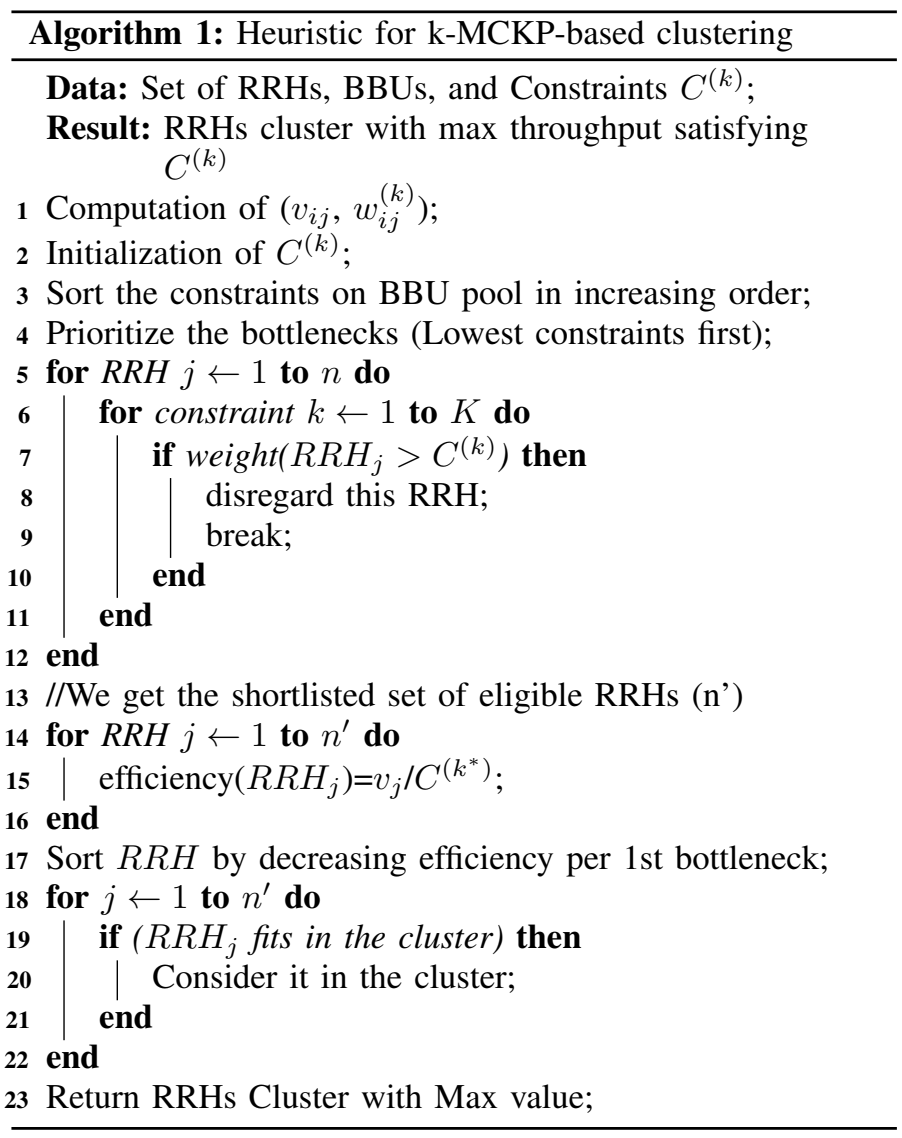

\section{Complexity of Optimal and Heuristic solutions}

The k-MCKP is one of the hardest variants of the Knapsack problem [6]. When input parameters are large (number of RRHs, number of BBU pools and number of weights dimensions), the problem becomes intractable. We used dynamic programming with tabulation method described in [6] to get the optimal solution. However, the order of complexity of the running time depends on the number of dimensions. For example, for $\mathrm{K}=2$, it is of order $O\left(K .(C[1])^{2} \cdot \max _{i \geq 2} C[i] \cdot n . m\right)$ where $\mathrm{n}$ is the number of items (that is the number of RRHs) and $\mathrm{m}$ is the maximum number of knapsacks (i.e., number of BBU pools), $\mathrm{C}[1]$ is the upper bound of the first constraint (i.e., outstanding bottleneck). For our heuristic, given that $\mathrm{K}$ is $\leq \mathrm{n}$, the sorting process is the time dominant task and its time complexity is $O(n . m . \log (n . m))$. 
TABLE I

SIMULATION PARAMETERS

\begin{tabular}{l|l}
\hline Parameter & Value \\
\hline Number of simulated RRHs & 12 \\
C1: Max No. of RRHs per BBU & 18 \\
C2: Max throughput per RRH & $130 \mathrm{Mbps}$ \\
C3: Max throughput per BBU & $1500 \mathrm{Mbps}$ \\
C4: Max No. of UE in an BBU & 10800 \\
C5: Data radio bearer per UE & 8 \\
White Noise Power density (N0) & $-174 \mathrm{dBm} / \mathrm{Hz}$ \\
Bandwidth (B) & $10 \mathrm{MHz}$ \\
Physical Resource Blocks & 50 \\
Cyclic Prefix & Normal \\
eNB Duplex Mode & TDD \\
PDSCH Tx Scheme & Tx Diversity \\
PDSCH Modulation & QPSK \\
Channel Delay Profile & Extended Vehicular A $($ EVA $)$ \\
Path loss Model & $92.45+20 \log _{10}(f)+20 \log _{10}(d)$ \\
Fading Model & Normal distribution $\mathcal{N}(0,1)$. \\
Transmit Antenna Gain & $8 \mathrm{dBi}$ \\
Poisson Parameter & $\lambda=1$ \\
PRB demand for UE & $\mathcal{U}(1,10)$ \\
\hline
\end{tabular}

\section{Performance Evaluation}

In this section, we report the performance of our k-MCKP based clustering approach by running Monte-Carlo simulation using Matlab. We start by describing the environment setup. Then, we analyze the results and discuss the effectiveness of our proposal compared to the basic strategies in C-RAN and the optimal solution. Two main scenarios are considered:

- No clustering versus k-MCKP RRHs Clustering.

- RRHs Clustering using our proposed heuristic and optimal solutions.

The simulation parameters are shown in Table I. Values of the constraints are chosen according to vendors' dimensioning rules in [7]. Without loss of generality; for the BBU pool, we consider $m$ to be " 5 ". Indeed, $C^{(1)}$ to $C^{(5)}$ correspond to the five constraints values on the BBU 3900 as per Huawei Datasheets of BBU 3900 [7]. The noise power density is assumed as constant with fixed value $N_{0}$. The rest of the parameters are for initializing the channel parameters for subframes simulation based on LTE channel model. They have unique effects on the values of the throughput and weights that are calculated in the first phase of our simulation.

Fig. 2 illustrates the effect of interference on the Physical Downlink Shared Channel (PDSCH) throughput for different values of interference expressed in term of SINR. In our setup, for an SINR bigger than $6 \mathrm{~dB}$, we see that the effect of interference on throughput is minimal and the throughput is barely affected, as depicted in Fig. 2(a). Such effect on throughput is expected as shown in equation (1). Consequently, we consider the SINR variation range from $-6 d B$ to $6 d B$. The channel capacity range is expected to be from 1 to $23 \mathrm{Mbps}$ according to this SINR range. When SINR increases, the effect of interference decreases and the achieved throughput increases.

Fig. 2(b) shows the Cumulative Distribution Function (CDF) of the normalized throughput. As anticipated, for a particular probability, normalized throughput is higher with SINR.

For illustration purpose, we considered a small network with 12 RRHs. Monte Carlo simulation is done with big number of experiments (10K). Performance is measured, displayed and plotted along to median and mean values. Estimation of the

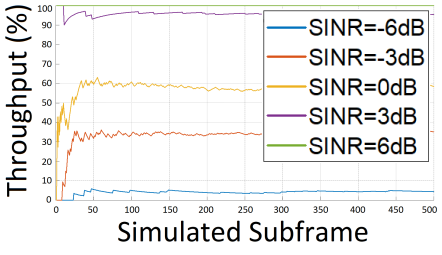

(a)

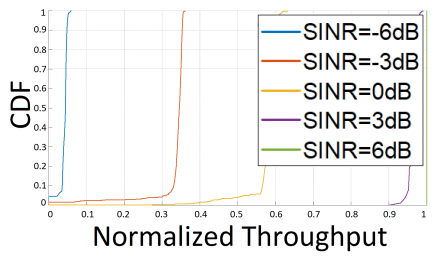

(b)
Fig. 2. Effect of Interference on PDSCH throughput for different SINR

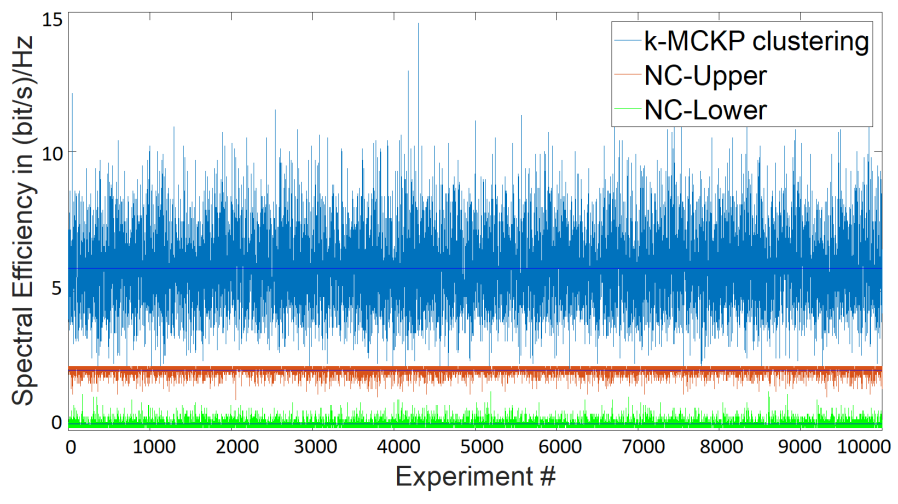

Fig. 3. Spectral Efficiency comparison (10K Experiments)

mean is done with $95 \%$ confidence level. Fig. 3 compares the spectral efficiency value contribution of our k-MCKP clustering approach with two No-Clustering approaches: Upper-bound (NC-Upper) and Lower-bound (NC-Lower). We can see that clustering provides higher spectral efficiency thanks to the use of joint transmission, where user data is received from multiple RRHs simultaneously in coherent manner. Specifically, we can observe from Fig. 3, that our k-MCKP approach enhances the spectral efficiency compared to the NC-Upper scheme.

Fig. 4 depicts the normalized frequency of occurrence of achieved throughput in $(b i t / s) / H z$ for the three schemes: k-MCKP, NC-Upper and NC-Lower. We can observe that the single RRH values are almost distributed from 1 to 2 (bit/sec)/Hz, whereas, considering our k-MCKP clustering approach, almost $80 \%$ of the throughput values lies between 4 and $8(\mathrm{bit} / \mathrm{s}) / \mathrm{Hz}$. This gain is due to the joint transmission effect within the RRHs cluster.

Fig. 5 further shows the gain of our proposed approach in terms of achieved throughput compared to the optimal solution as well as NC-Upper and NC-Lower schemes. We can see that our heuristic provides near optimal solutions. Indeed, as shown in Fig. 5, the gap is at most 10\%. This result is anticipated as our heuristic is a customized version of a greedy search approach that is known to be occasionally sub-optimal but much faster than the dynamic programming optimal solution as will be reported in Table II. In addition, we can see that our approach outperforms both NC-Upper and NC-Lower schemes. Specifically, the gain is at most $2(\mathrm{bit} / \mathrm{s}) / \mathrm{Hz}$ and $12(\mathrm{bit} / \mathrm{s}) / \mathrm{Hz}$, compared to NC-Upper and NC-Lower, respectively. Fig. 6 shows the impact of the number of RRHs on the average throughput for all the tested schemes. We can observe that the k-MCKP outperforms the no-clustering schemes, especially whenever the number of RRHs increases.

Finally, Table II reports the average computation times for 


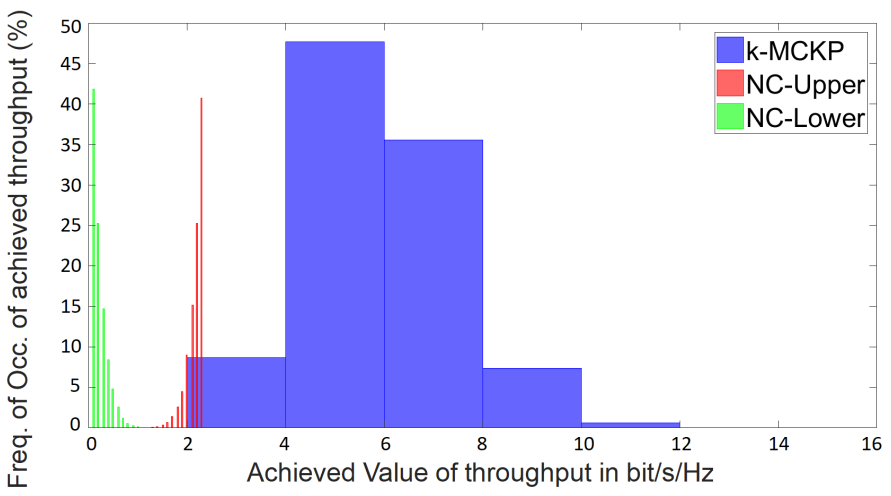

Fig. 4. Histogram of the throughput for both Cluster-RRH and Single-RRH

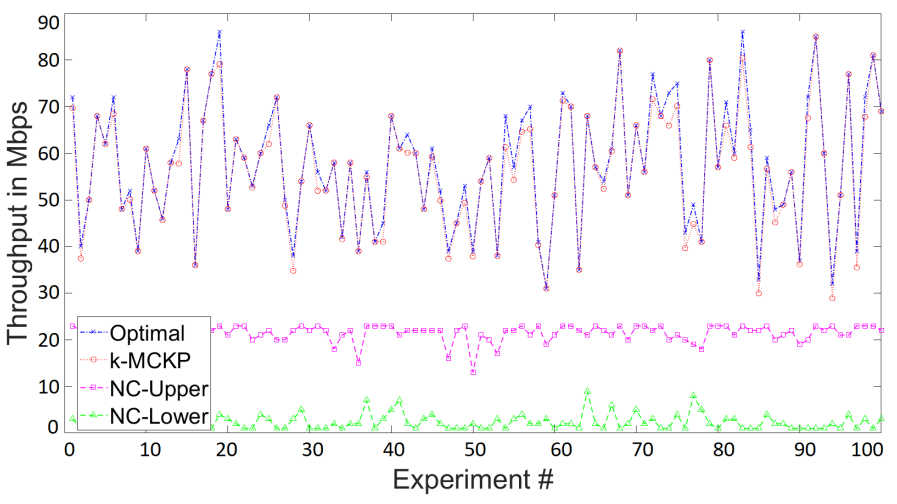

Fig. 5. Throughput in Mbps for optimal, heuristic and No-Clustering solutions

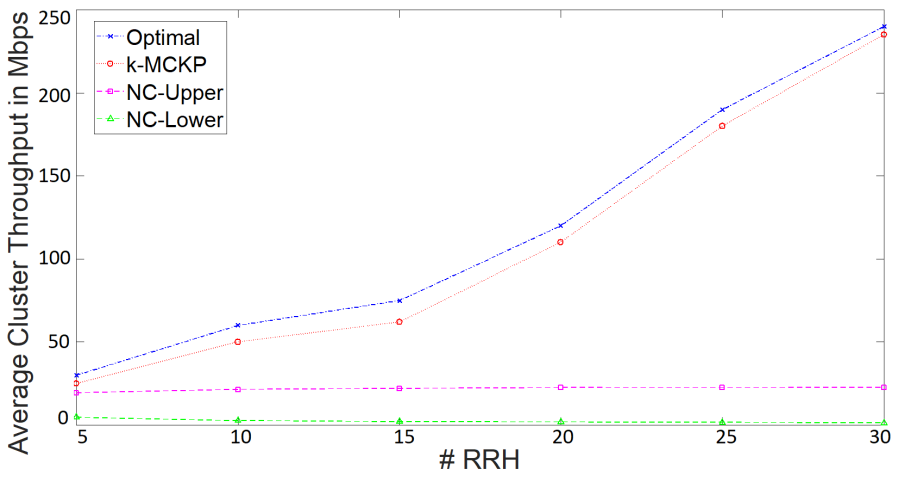

Fig. 6. Average throughput for all schemes

TABLE II

Average Computation Time (In MiLliseconds) FOR the Optimal AND THE PROPOSED HEURISTIC

\begin{tabular}{lllllll}
\hline Nb. of RRH & $\mathbf{5}$ & 10 & 15 & 20 & 25 & 30 \\
\hline Optimal scheme & 8.2 & 12.6 & 1390 & 14400 & 18650 & 24560 \\
k-MCKP Clustering & 2.87 & 3.32 & 13.2 & 21.63 & 54.23 & 96.2
\end{tabular}

the optimal and the proposed heuristic scheme. These measurements are performed on a PC with i7-7500U core with 2.7 GHz and $16 \mathrm{~GB}$ RAM. The reported results show that our proposed k-MCKP clustering approach takes a very short time to solve the problem (up to 96 Milliseconds when the number of RRHs is equal to 30), compared to the optimal one, which can reach 24,5 Seconds. This latter value is expected to increase in large-sized networks. Hence, according to Fig. 5 and Table
II, we can see that our proposed approach converges to the optimal solution and within a short time period, which makes it a feasible and efficient solution for C-RAN.

\section{CONCLUSION}

In this paper, we have addressed the throughput aware clustering of RRHs in C-RAN. We have formulated it as kMCKP and proposed a simple yet efficient heuristic to solve it. Results show that our proposal performs well compared to the optimal solution as well as the basic strategy in $\mathrm{C}$ RAN with no-clustering scenarios with obvious savings in terms of achieved throughput, spectral efficiency and processing time. As future work, we think of handling the problem using machine learning techniques.

\section{ACKNOWLEDGEMENT}

This work was partially supported by the FUI SCORPION project (Grant no. 17/00464), "Azm \& Saade Foundation”, and Lebanese University.

\section{REFERENCES}

[1] N. Salhab, S. El Falou, R. Rahim, S. E. El Ayoubi, and R. Langar, "Optimization of the implementation of network slicing in $5 \mathrm{~g}$ ran," in Communications Conference (MENACOMM), IEEE Middle East and North Africa. IEEE, 2018, pp. 1-6.

[2] M. Y. Lyazidi, N. Aitsaadi, and R. Langar, "Resource allocation and admission control in ofdma-based cloud-ran," in Global Communications Conference (GLOBECOM), 2016 IEEE. IEEE, 2016, pp. 1-6.

[3] K. Chen and R. Duan, "C-ran the road towards green ran," China Mobile Research Institute, white paper, vol. 2, 2011.

[4] E. Hernandez-Valencia, S. Izzo, and B. Polonsky, "How will nfv/sdn transform service provider opex?" IEEE Network, vol. 29, no. 3, 2015.

[5] "Physical channels and modulation (3gpp ts 36.211 version 12.4)," 2014.

[6] H. Kellerer, U. Pferschy, and D. Pisinger, "Knapsack problems," 2004.

[7] "Huawei technologies co. ltd, bbu3900 description."

[8] K. Boulos, M. El Helou, M. Ibrahim, K. Khawam, H. Sawaya, and S. Martin, "Interference-aware clustering in cloud radio access networks," in Cloud Networking (CloudNet), IEEE 6th International Conference. IEEE, 2017, pp. 1-6.

[9] H. Taleb, M. El Helou, K. Khawam, S. Lahoud, and S. Martin, "Centralized and distributed rrh clustering in cloud radio access networks," in Computers and Communications (ISCC). IEEE, 2017, pp. 1091-1097.

[10] K. Boulos, M. El Helou, K. Khawam, M. Ibrahim, S. Martin, and H. Sawaya, "Rrh clustering in cloud radio access networks with reassociation consideration," in Wireless Communications and Networking Conference (WCNC), 2018 IEEE. IEEE, 2018, pp. 1-6.

[11] M. Khan, R. S. Alhumaima, and H. S. Al-Raweshidy, "Qos-aware dynamic rrh allocation in a self-optimized cloud radio access network with rrh proximity constraint," IEEE Transactions on Network and Service Management, vol. 14, no. 3, pp. 730-744, 2017.

[12] M. Y. Lyazidi, N. Aitsaadi, and R. Langar, "Dynamic resource allocation for cloud-ran in lte with real-time bbu/rrh assignment," in Communications (ICC), 2016 IEEE International Conference. IEEE, 2016, pp. 1-6.

[13] M. Lahami, M. Krichen, M. Bouchakwa, and M. Jmaiel, "Using knapsack problem model to design a resource aware test architecture for adaptable and distributed systems," in IFIP International Conference on Testing Software and Systems. Springer, 2012, pp. 103-118.

[14] Y. Song, C. Zhang, and Y. Fang, "Multiple multidimensional knapsack problem and its applications in cognitive radio networks," in Military Communications Conference, 2008. MILCOM 2008. IEEE. IEEE, 2008.

[15] "3gpp, evolved universal terrestrial radio access (e-utra); further advancements for e-utra physical layer aspects, tr 36.814," 2010.

[16] S. Iqbal, M. F. Bari, and M. S. Rahman, "Solving the multi-dimensional multi-choice knapsack problem with the help of ants," in International Conference on Swarm Intelligence. Springer, 2010, pp. 312-323.

[17] M. Hifi, M. Michrafy, and A. Sbihi, "Heuristic algorithms for the multiple-choice multidimensional knapsack problem," Journal of the Operational Research Society, vol. 55, no. 12, pp. 1323-1332, 2004. 